\title{
Plaque-Psoriasis: Auch Dauertherapie mit Etanercept ist erfolgreich
}

- Die Zulassung von Etanercept $\left(\right.$ Enbrel $\left.^{\circledR}\right)$ bei erwachsenen Patienten mit mittelschwerer bis schwerer Plaque-Psoriasis ist erweitert worden: Außer einer intermittierenden Behandlung können sie jetzt auch eine Langzeittherapie über 24 Wochen hinaus erhalten. Sie erwies sich in einer 54 Wochen umfassenden Untersuchung als mindestens ebenso wirksam. Außerdem nahm unter beiden Therapievarianten die Lebensqualität erheblich zu.

Basis der Zulassungserweiterung sind die Daten von 720 erwachsenen Patienten mit mittelschwerer bis schwerer PlaquePsoriasis aus der CRYSTEL-Studie (Clinical randomized year-long study assessing the safety and efficacy of Enbrel in psoriasis). Darin erhielten die Probanden eine intermittierende Behandlung oder eine Dauertherapie. Die intermittierende Behandlung bestand aus $50 \mathrm{mg}$ Etanercept zweimal wöchentlich für 12 Wochen; bei Erreichen des Therapiezieles legten die Patienten bis zum nächsten Krankheitsschub eine Behandlungspause ein. Bei Dauertherapie wurden über die gesamte Studiendauer zweimal wöchentlich 25 mg Etanercept injiziert.
Beide Gruppen profitierten signifikant von ihrer Therapie, sagte Prof. Wolfram Sterry, Berlin. So verbesserte sich bei vergleichbarer Verträglichkeit z.B. die Krankheitsaktivität, ermittelt anhand des PASI-Wertes (Psoriasis Area and Severity Index), in der intermittierend behandelten Gruppe um mittlere $58,5 \%$ (Grafik 1). Bei Patienten mit Dauertherapie war der Nutzen mit 67,5\% noch größer. Die intermittierende Behandlung erforderte dabei ein Viertel weniger Wirkstoff. Auch der PGA-Wert (Physician's Static Global Assessment of Psoriasis) sank damit stärker ab, nämlich auf 1,89 von 5 Punkten (Vergleichsgruppe: 2,61). Nagelbefall und Gelenkbeschwerden wurden durch die Dauertherapie ebenfalls günstiger beeinflusst: Der Nagelbefall ging um 56,5\% zurück und die Gelenkbeschwerden um 46,3\%. Die intermittierende Therapie erzielte einen Rückgang um $43,5 \%$ und $35,3 \%$.

Gleichzeitig verbesserte sich auch der dermatologische Index der Lebensqualität (DLOI), so Dr. Sandra Philipp, Berlin. Er kann bis zu 30 Punkte erreichen; mehr als 10 Punkte zeigen eine deutlich eingeschränkte Lebensqualität an, wie sie bei Psoriasis häufig ist. Das bestätigte sich auch zu Beginn

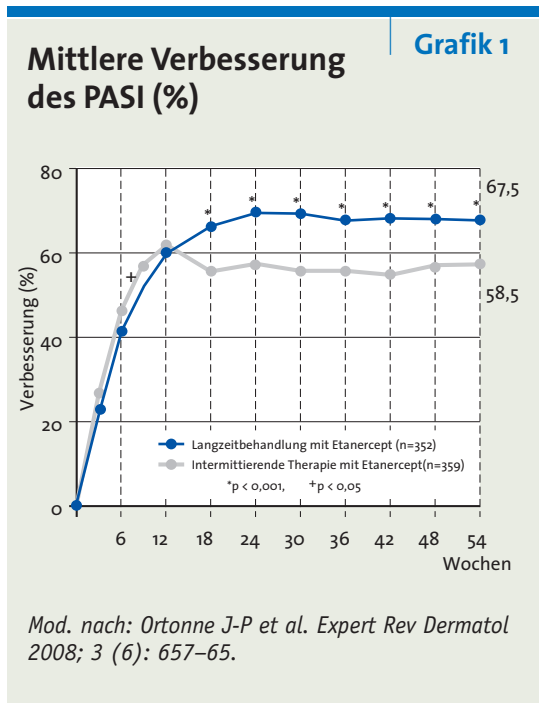

von CRYSTEL - da lagen die Werte bei 12,8 und 13,8 Punkten. Die Dauertherapie verbesserte sie um $69,5 \%$, die intermittierende Therapie erzielte 55,3\%. Entsprechend waren die meisten Patienten mit ihrer Behandlung zufrieden - über die gesamte Studiendauer hinweg.

hb

Pressekonferenz „Chrystel zeigt positive Daten zur Langzeitbehandlung mit Etanercept bei Plaque-Psoriasis". Im Rahmen der 18. EADV-Tagung, Berlin, 8.10.2009 (Veranstalter: Wyeth).

\section{„Physiologisches“ Anti-Aging für die Haut ab 60}

— Die Haut verändert sich mit zunehmendem Alter in mehrfacher Hinsicht: Der Gehalt an essenziellen Fettsäuren (insbesondere Linolsäure) nimmt ab. Auch kollagene Fasern und Hyaluronsäure sind reduziert. Mit Avène Sérénage wird ab Januar 2010 ein neues Anti-Aging-Produkt zur Verfügung stehen, das speziell für Frauen im Alter ab 6o Jahren entwickelt worden ist. In der Creme ist Glycoleol ${ }^{\circledR}$ enthalten, eine Vorstufe der Linolsäure, das als Depot wirkt. In der Haut erfolgt enzymatisch eine kontinuierliche Metabolisierung zu Linolsäure. Dadurch wird der Säureschutzmantel der Haut konstant unterstützt und der epidermale Wasserverlust vermindert. Ein weiterer Bestandteil ist H.A.F ${ }^{\circledR}$. Diese HyaluronsäureFragmente sind in der Lage, tief in die Haut einzudringen. Die Fragmente induzieren auch die zelleigene Produktion von Hyaluronsäure und von Kollagen. Die Wasseraufnahmekapazität der Haut nimmt zu, das kollagene Stützgewebe wird regeneriert und nimmt an Dichte zu.

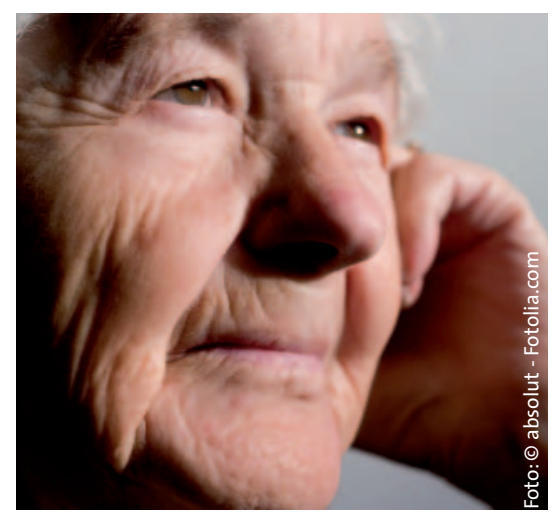

Ein weiterer Wirkstoff ist Pro-Tocopherol ${ }^{\circledR}$, die einzige stabile und leicht von der Haut assimilierbare Form von Vitamin E. Auch diese Substanz wirkt als Depot, das nach Bedarf in aktives Tocopherol umgewandelt werden kann. So entsteht ein kontinuierlicher Schutz gegen freie Radikale und damit gegen die nachteiligen Effekte von UV-Strahlen oder weiteren belastenden Umweltfaktoren auf die Haut.

Die neue Öl-in-Wasser-Emulsion enthält keine Parabene, ist hypoallergen und nicht komedogen. Sie enthält zu 52\% Thermalwasser mit niedrigem Anteil an Mineralstoffen und hoher Konzentration an Kieselerde.

af

Pierre Fabre Infotag. München, 5.11.2009 (Veranstalter: Pierre Fabre Dermo-Kosmetik). 